\title{
The Enhances Effect of VR on Awareness of Environmental Protection
}

\author{
Yinan $\mathrm{Xu}^{1, *}$ \\ ${ }^{1}$ College of Science and Technology, Wenzhou-Kean University, Wenzhou City, Zhejiang Province, China, 325000 \\ *Corresponding author.Email: yinanx@kean.edu
}

\begin{abstract}
The development of science and technology makes people begin to use more advanced technologies to alleviate environmental problems. However, research and technology applications for establishing and enhancing environmental awareness require huge costs and technical support. Even though there are eco-themed games in current game market, including virtual reality games, they have no significant effects. In order to figure out whether playing virtual reality game related to environmental protection enhances player's environmental awareness or not, this paper analyzes different kinds of eco-themed games and their effects by comparing the secondary data, and the paper also demonstrates a game vision. It concludes that playing virtual reality games related to environmental protection has immediate and delayed effects on people's awareness.
\end{abstract}

Keywords : Virtual Reality Game, Habit, Effect, Environmental Protection, Awareness

\section{INTRODUCTION}

Along with the rapid development of technology, the appearance of augmented reality and virtual reality allow users to perceive the world and understand a concept via a unique angle and a novel method. Although technology improves people's life quality in various aspects, such as education, medical treatment, and transportation, humans still need to put much more effort into applying technology to the environment. In other words, environmental issues which continuously accompany the progress of the civilization need the involvement of technology.

Nowadays, companies put some products into use are invented to remit environmental problems. For example, electric cars reduce the emission of automobile exhaust, and biodegradable material declines soil pollution. Meanwhile, enhancing an individual's awareness of environmental protection is a fundamental approach to alleviating environmental pressure. However, the combination of virtual reality and environmental protection is immature in daily life, even in researches. This paper explores the effect of playing virtual reality games, a widely accepted form, on consumers' awareness of environmental protection. By comparing the current games, experiences, or exhibitions related to the environment, the paper demonstrates a possible and practical VR game to promote player's awareness of environmental protection. In this way, users form an environmentally friendly habit and behave it in reality.

\section{ANALYSIS}

This section criticizes a traditional publicity stunt and evaluates games related to environmental issues in the current VR game market through multiple factors such as downloads, user experience, flaws, and effects.

\subsection{Traditional Publicity Stunt - Poster}

As a traditional publicity stunt, posters are usually posted on roadside walls, billboards, and underground tunnels to call for environmental protection. However, it is a huge price to pay for what it does offer. Both the design and the publishing of the poster cost much money. The size and the printed material of the poster also affect the price. Moreover, it usually takes around 20 minutes to print a poster and about 14 days to print 1000 posters. Then, the government purchases a place and employs workers to post the poster. The poster adds the financial burden to the government's tight budget and intensifies the environmental problem. In other words, citizens cut down trees to make paper and then print it to be a poster, and the production of the poster involves the consumption of fuel to provide energy. 
On the other hand, the environmental protection poster hardly changes people's minds since these posters are static with limited content. In most cases, these posters seldom arise people's attention and are ignored by passengers. Therefore, using posters, newspapers, and pamphlets to advocate environmental protection is expensive and inefficient. Society needs a solution with effectiveness and sustainability to enhance the public's environmental awareness.

\subsection{Virtual Reality Games}

Virtual reality games offer a high immersive environment for the game players and facilitate the interaction between humans and computers to present the concept or idea of designing a specific game. According to Cailianshe, the global VR industrial application market in 2019 exceeded 400 million, with a growth rate of $145 \%$. It is estimated that the VR industrial market will reach 3.36 billion in 2023 [1]. In 2019, there were about 10 million virtual reality consoles around the world [1]. However, compared with about 240 million home video game consoles worldwide, virtual reality games are in their early stages of development, and the market's potential is enormous. Playing virtual reality games, players obtain an immersive experience using their multiple senses, surrounded by a 360 -degree gaming environment. Also, what the player acts, especially the body movement, is synchronized with the action of the player's game character. After contrasting sales of various virtual reality games, whether a virtual reality game could stand out and become popular largely depends on its playability instead of the fidelity of the picture. In other words, even though the virtual reality game is designed with a cartoon style, the game is welcomed if it is friendly to players, including interaction, simplicity, rhythm, and synchronization.

\subsection{Games Related to Environmental Protection}

Doomsday Vault is a kind of adventure puzzle-solving game. Developers design the game with a meaningful theme that is finding and preserving precious seeds to grow crops. Furthermore, the game concentrates on offering players diverse puzzles and an amazing virtual world to explore. However, the relationship between the fun of the game and the environmental protection theme is weak. Players are more likely to focus on solving puzzles and exploring the world.

Compared with Doomsday Vault, Eco clicker contributes more to achieving the goal of promoting environmental protection. Eco clicker is a game where players can click the screen to make the game character pick up the garbage to recover the polluted earth. The more clicks a player has, the faster the character picks up. This game presents the idea of environmental protection through clicking on such an interaction model. Most of the nearly 30,000 downloads users gave good reviews because the game is easy to understand and manipulate. However, clicking is the only way to manipulate the game, which seems to be toneless, and we cannot protect the environment in reality by clicking. In other words, consumers hardly know how to do specific things to protect the environment through this game.

Another game called Refuse Classification asks players to throw various kinds of garbage into the correct classified trash bin to throw the garbage in the correct trash bin in real life. One point that distinguishes Refuse Classification from the other two games mentioned above is that this game is a virtual reality game. Although this game has been on Steam for several years, it has few downloads. The possible reason is that the game lacks incentives to maintain player's interest, and the game is too dull since it is designed only for garbage classification. Moreover, applying virtual reality to the game seems inappropriate because the game does not fully use the immersion and synchronization of a virtual reality game.

\subsection{Possible Effects on Environmental Protection}

According to Yee, Bailenson, and Ducheneaut, the Proteus effect refers to a phenomenon that is users infer from their avatars' appearance around their expected characteristics and then conform to these behaviors offline [2]. The study demonstrates the Proteus effect in immersive virtual reality, which will persist in real-life face-to-face interactions. However, the study only focuses on the appearance of the avatars instead of the avatar's behaviors, so further studies need to show that the avatar's behavior will persist in subsequent interactions in reality. Meanwhile, behavioral transforming requires a progression via a series of periods [3].

Some research was with a similar topic that uses virtual reality to enhance behavior intention. Hsu, Tseng, and Kang experimented that participants go through exaggerated feedback in virtual reality for water conservation [4]. They conclude that the feedback brings about two types of effects which are immediate effects and delayed effects. In other words, the participants realize that they are wasting water and improving it in virtual reality, but they also contribute to water conservation in real life. In addition, the experiment made by Nelson, Anggraini, and Schluter indicates that participants who experience the virtual reality of environmental conservation donate more to a conservation charity organization than participants who have just received a written donation request [5]. Nonetheless, the degree of effects from virtual reality 
depends on complex variables, such as immersive experience, target population, and the length of the experience. It is worth more research about the effect of virtual reality on human behavior and intention.

\section{DISCUSSION}

This section explains a new idea of applying virtual reality games to enhance player's behavior intention on environmental protection and discusses the application and deficiency of the current virtual reality devices for this idea.

\subsection{Game Vision}

Figure 1 indicates that the player, for example, initially lives on the side of a polluted lake at the beginning of the immersive virtual reality.

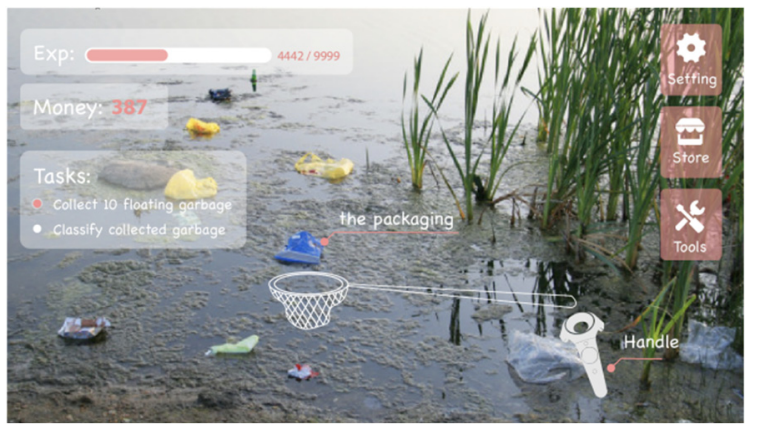

Figure1: Game Interface

A novice will be assigned a series of tasks to save the polluted lake. For example, the player needs to fish out the trash floating in the lake with a net. Then the user will follow the correct garbage classification to dispose of the collected garbage. In this way, tasks motivate players to continuously play this game and get an immediate sense of accomplishment. Additionally, the player understands how to solve the environmental pollution, especially the difficulty of processing the pollution. At the same time, the game is becoming active to inform players and spark player's interest. To be more specific, if the player does not finish the tasks within a certain period, the player will experience aquatic animals eating the floating trash and water weeds entangle the garbage. After the lake is improved, the player can obtain economic benefits in virtual reality through various ways, like selling fish. The player could invest in protecting and improving the lake environment. For instance, the player can grow trees along the lake to purify the water sources.

As the player's experience accumulates, the player will gradually gain the opportunity to open up land to protect more contaminated areas, which is the unlocking mechanism. This mechanism satisfies the player's curiosity and evokes the client's enthusiasm towards environmental protection. The project will make users experience how to save a polluted area in immersive virtual reality and understand the meaning of protecting the environment after feeling the difference between a polluted area and a protected area. After forming a good habit in a virtual reality game, the player possibly behaves in real life, which refers to a delayed or long-term effect.

\subsection{Application and Constrains of Technology on Virtual Reality game}

The player could see the polluted lake by wearing virtual reality glasses and smell the stench of the polluted lake via the architecture of scent. However, the progress of olfactory technology is slow, partly resulting from the difficulty of storing odors. Also, whether a player accepts or favors the odor requires much more researches and experiments. In addition, background music and sound effects are two crucial elements that affect the immersion and the gaming experience [6]. Thus, this project also involves the user's auditory sense to promote the gaming experience.

Although virtual reality offers a three-dimensional immersive experience and a high resolution for players, it quickly causes motion sickness so that users are too tired to experience the game for long periods or even cannot adapt to the virtual reality game at first. At this point, the market for virtual reality dramatically shrunk. Patrao, Pedro, and Menezes propose that keeping the immersion from the start until the end of the experience to respond to the user whenever they request it could reduce motion sickness [7].

Moreover, based on the idea of behavior synchronization of humans and robots, the project can give a sense of accomplishment to the player [8]. In other words, what the player does in virtual reality will be realized by the robot in reality at the same time. At this point, though the player may not be able to save a lake in real life, the player contributes to the protection of the lake.

\subsection{Summary}

To summarize, the project offers an immersive virtual reality to users, and users can experience the participation of protection to understand the protection process and enhance their awareness of environmental protection. Instead of thinking that environmental protection is far from us, everyone could contribute to it.

\section{CONCLUSION}

Compared with traditional ways like posters mentioned above, eco-themed virtual reality games provide a more effective solution to form a habit and enhance environmental protection awareness. Researches show that applying virtual reality to 
environmental protection has both immediate and delayed effects. In other words, users are likely to behave what they have learned in virtual reality. If they have experienced a polluted environment and have done a series of processes to deal with pollution, they can understand the meaning of protecting the environment and enhancing environmental protection awareness.

Even though some games, including virtual reality games, are designed with an environmental theme, they seldom effectively promote awareness of environmental protection. This paper proposes a game vision, which combines immersion, interaction, and education. Whether this game vision effectively enhances awareness of environmental protection and the degree of the effects needs further experiments and is implemented to be on the ground in the future. Moreover, figuring out the other elements that possibly affect the effects of eco-themed virtual reality games. To be more specific, the immersion experience, the time, and the user-friendliness usually influence on the extent of effects. A future study needs to be done to verify it.

\section{REFERENCES}

[1] Cailianshe, (2019), In 2019, the global AR/VR industrial application market scale increased by $145 \%$ http://www.cls.cn/detail/418669

[2] Yee, N., Bailenson, J. N., \& Ducheneaut, N. (2009). The Proteus effect: Implications of transformed digital self-representation on online and offline behavior. Communication Research, 36(2), 285-312.

[3] Riva, G., Banos, R. M., Botella, C., Mantovani, F., \& Gaggioli, A. (2016). Transforming experience: the potential of augmented reality and virtual reality for enhancing personal and clinical change. Frontiers in psychiatry, 7, 164.

[4] Hsu, W. C., Tseng, C. M., \& Kang, S. C. (2018). Using exaggerated feedback in a virtual reality environment to enhance behavior intention of water-conservation. Journal of Educational Technology \& Society, 21(4), 187-203.

[5] Nelson, K. M., Anggraini, E., \& Schlüter, A. (2020). Virtual reality as a tool for environmental conservation and fundraising. Plos one, 15(4), e0223631.

[6] Gormanley, S. (2013). Audio immersion in games - a case study using an online game with background music and sound effects. The Computer Games Journal, 2(2), 103-124.

[7] Patrão, B., Pedro, S., \& Menezes, P. (2020). How to deal with motion sickness in virtual reality.
[8] Igorevich, R. R., Ismoilovich, E. P., \& Min, D. (2011, November). Behavioral synchronization of human and humanoid robot. In 2011 8th International Conference on Ubiquitous Robots and Ambient Intelligence (URAI) (pp. 655-660). IEEE. 